\title{
Conocimiento y estructura en la investigación académica: una aproximación desde el análisis de redes sociales
}

\author{
José Manuel Gaete Fiscella - Departamento de Sociología (U. de Salamanca,
} España) y J orge Ignacio Vásquez - Departamento de Sociología (U. de Chile)

\begin{abstract}
Resumen
El presente artículo se presenta como un aporte al enfoque de redes sociales en el campo de la educación superior, a partir de la identificación y análisis de la(s) red(es) de conocimiento recreada(s) a partir de dinámicas relacionales que establecen los investigadores ligados al desarrollo de las ciencias sociales y grupos de investigación en el devenir de la actividad investigadora.
\end{abstract}

Consiste en un estudio de carácter reticular/ cuantitativo al interior de un departamento académico[1]. Desde el enfoque analítico de la teoría de redes se estudia la generación de conocimiento en redes informales de académicos, poniendo el énfasis en la importancia de las relaciones interpersonales para la transmisión del conocimiento y la reproducción del capital intelectual, y la correspondiente diferenciación de posiciones en el espacio social académico.

El texto se estructura en tres partes. En la primera, se realiza una revisión de los principales lineamientos teóricos referentes al tema, tales como sociedad del conocimiento, nuevas tendencias en la sociología organizacional, las diversas corrientes relativas al capital social y análisis de redes. En una segunda etapa se llevan a cabo pruebas de hipótesis y tratamiento de datos reticulares, la correspondiente generación de indicadores de estructura y centralidad de red, para finalmente, compartir las conclusiones a partir de los datos y la complementación de técnicas estadísticas.

Palabras clave: capital social - sociedad del conocimiento - redes sociales - redes de expertos - colegios invisibles - subestructuras de red.

\begin{abstract}
The present article appears as a contribution to the approach of social networks in the field of the high education according to the identification and analyses of "networks of knowledge" recreated from the relationships establish between investigators related to the development of social sciences and groups of mutual support, in the context of the investigation activity.
\end{abstract}

Consist in a "quantitative- reticular" study in the interior of a study department[2] with structured surveys. Our analytical approach it is from the networks theory, and the generation of knowledge in informal organizations of academic studies, putting the emphasis in the importance of the interpersonal relationships for the transmission of the knowledge and the reproduction of the intellectual capital with the corresponding differentiation of positions in the academic social space.

The text is structure in three parts. First a revision of the main referring theoretical headlines to the subject is made, such as society of the knowledge, new tendencies in organizacional sociology, the diverse currents relative to the share capital and analysis of network. Secondly, it is carried out a test of hypothesis and reticular data processing, the generation of indicators such as structure and centrality of network, and finally, whe share the conclusions from the data and the complementación of statistical techniques.

Key words: social capital - society of the knowledge - social networks - networks of experts - invisible schools - network substructures. 


\section{I.a La asociatividad para la generación de conocimiento, diferenciación en los espacios formales de investigación.}

Definir y explicar lo que se entiende por conocimiento no es una tarea trivial, y muchas veces se confunde con el concepto de información. Si bien son conceptos distintos, la relación entre ambos es muy importante para el proceso de aprendizaje, específicamente el organizacional. Así pues, ambos son recursos que se caracterizan por pertenecer a un contexto específico y ser relacionales, en el sentido que dependen del entorno y se crean dinámicamente fruto de la interacción social (Nonaka y Takeuchi, 1995). Berger y Luckman (1966) establecen que las personas que interactúan en un cierto contexto histórico y social comparten información y construyen conocimiento, el cual, influye en sus juicios, comportamientos y actitudes. El conocimiento a diferencia de la información se refiere a la acción, y es inseparable del pensamiento. Por ello, el conocimiento impregna datos e información con decisiones y acciones relevantes (Fahey y Prusak, 1998), de tal manera que se crea conocimiento a partir de la información (flujo de mensajes) anclada en las creencias y compromisos que el individuo posee (Nonaka y Takeuchi, 1995, en Zárraga, C.M. 2003).

Una primera definición de la generación de conocimiento en las organizaciones se puede entender según Caraballo (2006) como el proceso que amplifica organizativamente el conocimiento creado por los individuos y que se cristaliza como una porción de la red de conocimiento de la organización (Nonaka y Takeuchi, 1995). Así, el conocimiento organizacional no proviene exclusivamente de la sumatoria de las dotaciones de conocimiento individuales de todos y cada uno de los miembros de la organización (Spender, 1996; Nonaka y Takeuchi, 1995), por cuanto existe la necesidad de compartir estos conocimientos con el resto de los partícipes de la misma, e integrarlos en el conjunto de las tareas, de las funciones y de las actividades de la organización.

Pero además de las formas de generación de conocimiento nos interesa tener en cuenta que existen diversas formas de reproducción, transmisión y difusión del conocimiento. Así por ejemplo para Julve (2000) el intercambio de conocimientos explícitos y generales puede realizarse, bien mediante medios de comunicación impersonales, o bien mediante canales formales. Por el contrario, los conocimientos tácitos y particulares sólo pueden ser transmitidos lentamente y con dificultad, mediante la interacción personal, estrecha e interesada de los individuos que lo poseen. Es por ello, que los mecanismos para tener acceso, para poder integrar, y para generar interés en el intercambio de este tipo de conocimientos deben tener un carácter informal o social. 
REDES- Revista hispana para el análisis de redes sociales

Vol.14,\#5, J unio 2008

http://revista-redes.rediris.es

De igual manera, Rosalba (2003) resalta la importancia que tienen las interacciones personales en la transmisión del conocimiento. Es aquí donde el concepto de flujos de conocimiento se integra con el concepto de redes. Éstas adquieren mayor importancia cuando se está tratando de localizar las fuentes de conocimiento tácito. La interacción personal y la movilidad son los únicos canales a través de los cuáles se obtiene el conocimiento tácito. Por ello Senker y Faulkner (1996) plantean que el conocimiento tácito es un elemento muy importante del conocimiento transferido mediante las redes personales.

Como una primera aproximación conceptual sobre el conocimiento, su producción y transmisión, se puede afirmar que el conocimiento, además de ser un factor capital para las organizaciones, es un factor muy complejo tanto en su creación como en su socialización, por ello, se plantean ciertas prácticas o directrices organizacionales que permitan facilitar su capitalización. Esta forma de administrar el conocimiento es lo que se ha definido en la literatura como "Gestión del Conocimiento" y según Caraballo (2006) sus principales directrices son:

a. Actividades destinadas a la formación de los individuos para la adquisición de nuevos conocimientos.

b. Creación de grupos y su capacitación en habilidades grupales.

c. Implantación de mecanismos que abarquen la totalidad de la información (interna y externa) y lleguen a todos los miembros de la organización de una forma rápida y precisa.

d. Compromiso expreso de la dirección en la institucionalización del conocimiento en rutinas organizativas.

e. La socialización en una cultura y valores comunes que fomente generar y compartir conocimientos, es decir, que proporcione a sus miembros las actitudes necesarias para querer y poder aprender.

En la actualidad resulta casi indiscutido que para toda organización uno de los capitales más importantes es el conocimiento. Por tanto, la Universidad y sus organismos departamentales no escapan a esta realidad, al contrario, precisamente son estas organizaciones las que por excelencia se espera tengan como función primordial la creación de conocimiento, plasmado principalmente en el desarrollo de los llamados "proyectos de investigación".

En dicho ámbito, no es común que un investigador por sí sólo ejecute un proyecto de investigación, por lo general, estos proyectos son llevados a cabo por "grupos de investigación", definidos como equipos de investigadores más o menos formales, 
REDES- Revista hispana para el análisis de redes sociales

Vol.14,\#5, J unio 2008

http://revista-redes.rediris.es

de composición más o menos variable y comúnmente dirigidos por un "investigador principal". Si consideramos que las organizaciones hoy en día buscan nuevas formas de organizarse, nuevas estructuras funcionales con la finalidad de tener una mayor flexibilidad para enfrentar el elevado grado de incertidumbre y demandas cambiantes del entorno, no es de extrañar que sean estas instancias, (los grupos de trabajo[3]), las estructuras que permiten ganar mayor flexibilidad para acomodarse a las nuevas circunstancias del entorno.

Para Solís (2004) la forma tradicional de organización de las comunidades académicas se define en la agrupación por disciplinas científicas especializadas que son fuente de "membresía, unidad, prestigio y autoridad" (en Clark, 1984), y son estructuradas jerárquicamente para definir y establecer problemas, métodos y normas cognitivas en la creación de conocimiento científico, cuya calidad es controlada por la propia evaluación de los pares (Gibons et al., 1997). Según investigaciones como la de Clark (1995) se revela que las naciones con mayor avance en educación superior en el mundo occidental y asiático (Francia, Estados Unidos, Alemania, Inglaterra y Japón) parten de ciertos arreglos organizacionales en sus sistemas universitarios que buscan favorecer de manera vigorosa la investigación como base para la enseñanza y el estudio. En el nivel de las unidades básicas se busca vincular de manera estrecha y efectiva la enseñanza y el aprendizaje con los grupos de investigación como los elementos claves, en cuyo seno los participantes son sometidos a un flujo de conocimientos. Por tanto, los grupos de investigación transmiten el conocimiento tácito, y los grupos de docencia propagan el conocimiento explícito (Suárez, 2006).

Como su nombre lo indica, los grupos de investigación son una parte o sección de la organización, lo que por definición implica que existirán miembros excluidos total o parcialmente de estas instancias, y por ende, de la producción de conocimiento e información que en ellos se genere. Por el contrario, habrán otros que tendrán posiciones más relevantes, influyentes o importantes en la dinámica investigadora, ya sea por relaciones de estatus profesional y experiencia; la cantidad de proyectos que ejecutan; la cantidad de investigadores que implican o agrupan; la cualidad y cantidad de publicaciones; recursos que logran concursar, entre otros factores que paulatinamente van generando un diferencial material y simbólico dentro de un cuerpo de investigadores.

A su vez, no escapa a esta realidad el que una de las características fundamentales de los grupos de investigación sea el hecho que los investigadores que lo componen pueden, y generalmente así lo hacen, participar paralelamente en otros proyectos 
REDES- Revista hispana para el análisis de redes sociales

Vol.14,\#5, Junio 2008

http: // revista-redes.rediris.es

de investigación. Dicha acción da pie para contactar y conectar a distintos grupos entre sí, o en su menor grado, unir a investigadores de forma indirecta con ciertos grupos de investigación. De esta manera, se pueden observar diversas estructuras reticulares, como por ejemplo una red de relaciones laborales, que además, se entiende como una red de información y conocimiento, en la medida en que puede existir transmisión de información entre los distintos investigadores pertenecientes a distintos grupos de investigación, y aún más, cuando los distintos proyectos son variantes de un área de investigación específica. En consecuencia, es en estos contextos relacionales donde se generan nuevos posicionamientos de influencia y de poder sobre el flujo de información y conocimiento, roles que pueden ser muy distintos a los posicionamientos tradicionales de poder y decisión fundados en atributos como las categorías profesionales o académicas.

En función de lo anterior, nuestro estudio de caso consiste en aplicar un análisis de redes sociales a las distintas relaciones que puedan existir, intra y entre, los grupos de investigación que se conforman en el interior de un determinado departamento académico y de investigación. Si bien los resultados no son generalizables para otras comunidades de investigadores, sí esperamos dilucidar de que manera el análisis de redes nos permite identificar y caracterizar a los investigadores que desarrollan posiciones de poder o influencia (relacional) dentro de una determinada red académica, y explorar en qué medida dicha posición puede (o no) estar determinada por el cargo o categoría profesional que el individuo desempeña dentro de un determinado departamento[4].

\section{I.b Capital social y redes, influencia y poder en las relaciones sociales para la generación de conocimiento}

Formalmente, uno podría esperar que los departamentos académicos de las instituciones de educación sean las encargadas de organizar y desarrollar la investigación y las asignaturas propias de su área de conocimiento, pero en la práctica, muchas veces la unidad de investigación en la universidad es el grupo de investigación y no el departamento. Por lo general, el departamento se considera una unidad de carácter administrativo, en ocasiones con un tamaño muy grande, en la que conviven grupos que desarrollan líneas diferentes o divergentes de investigación. En este sentido, algunos investigadores consideran que la falta de contactos entre los diferentes grupos de un mismo departamento hace que no se aprovechen debidamente las sinergias que se podrían dar entre ellos (Olazaran, 2004). 
REDES- Revista hispana para el análisis de redes sociales

Vol.14,\#5, J unio 2008

http://revista-redes.rediris.es

En consecuencia, Solís (2004) estima que las universidades y los centros de investigación para poder participar en los circuitos de conocimiento requieren de una capacidad para redimensionar sus fronteras organizacionales, y además, adquirir la competencia en el diseño de formas organizacionales en red.

Las redes de conocimiento presentan en su operación distintos modelos de colaboración, y muchas veces muestran combinaciones de dichos modelos. Una tipología de estos modelos de colaboración es presentada por Creech (2001, cit. en Solís, 2004), a partir del espectro de sus intereses y de su ubicación, ya sea en el contexto interno de una sola organización o en el contexto externo de múltiples organizaciones:

a) Redes de gestión de conocimiento interno. Para maximizar la aplicación del conocimiento individual a los objetivos de la organización, estas redes evolucionan a través del mapeo temático de la experiencia dentro de la organización, y de ambientes propicios para compartir el conocimiento.

b) Alianzas estratégicas. Para obtener una ventaja competitiva sustantiva y reforzar su lugar en el mercado estas redes establecen una colaboración de largo plazo entre organizaciones asociadas.

c) Comunidades de práctica profesional. Con el deseo de reforzar competencias personales, estas redes informales y voluntarias se conforman con dos o más individuos, en espacios de conversación e intercambio de información que guían el posible desarrollo de nuevas ideas y procesos.

d) Redes de expertos. Para promover estratégicamente hallazgos de investigación hacia instituciones seleccionadas como claves. Estas redes reúnen bajo invitación a expertos reconocidos de un campo en reuniones e interacción electrónica regulares.

e) Redes de información. Para dar acceso a información proporcionada por los miembros de la red, estas redes abiertas vinculan usuarios (individuos $u$ organizaciones) de manera electrónica en información usualmente ordenada por contenido temático.

f) Redes de conocimientos formales. Con el propósito de influir en los individuos o en los grupos tomadores de decisión, estas redes formales apoyadas institucionalmente, reúnen expertos en temáticas específicas y comprometidos con objetivos y programas de trabajo bien definidos sujetos a evaluación. Las redes de conocimiento formales representan el modelo organizacional más cercano a los 
REDES- Revista hispana para el análisis de redes sociales

Vol.14,\#5, J unio 2008

http://revista-redes.rediris.es

requerimientos de coproducción de un conocimiento útil con valor económico y de formación de recursos humanos, en razón de que la institucionalización de estos vínculos permite desarrollar programas de largo plazo.

En esta línea es de esperar que las elites investigadoras sean mayoritariamente conservadoras, en cuanto a abrir nuevas áreas de investigación o conocimiento, ya que de esta forma logran mantener su poder y control sobre las actividades y temas de investigación. Por otra parte, los investigadores más noveles y con menos poder tienden a relacionarse con otros grupos de investigación (e investigadores) como modo de burlar la tendencia conservadora de las elites científicas. En este contexto Mulkay vincula la innovación intelectual en la ciencia a la movilidad de los científicos de un área de investigación a otra.

La estabilidad de las líneas de investigación, en torno a una elite definida, puede dar lugar o ser indicio de lo que en sociología de la ciencia se conoce como "Colegio Invisible". Se denomina así al círculo de personas que desarrollan su actividad investigadora bajo la influencia de un líder con un reconocido prestigio y una elevada productividad científica. En torno a ellos se establece una red de comunicación tanto formal como informal, donde los integrantes establecen un círculo social en el que no necesariamente se conocen de forma personal entre ellos (Crane, 1972).

En un principio este tipo de organizaciones se definieron en torno a la coautoría de publicaciones científicas, sin embargo, Derek J. de Solla Price en Science Since Babilón (1961) utiliza la expresión de "nuevos colegios Invisibles"[5] para definir a los grupos de relaciones informales entre científicos e investigadores fuera de las relaciones formales de coautorías científicas y académicas. Dichas estructuras utilizan como vehículo de comunicación los llamados documentos "preprints", o artículos científicos en fase de elaboración, que se intercambian para su revisión y comentarios, generando nuevas redes como estructuras fundamentales en la producción científica (Gracia, 2005).

Por lo tanto, lo fundamental es distinguir que la existencia de un "Colegio Invisible" hace referencia a un "círculo social" en el que los integrantes generalmente conocen sólo a una parte de dicho círculo o red, pero que igualmente son influidos por los otros integrantes con los que no están conectados directamente[6].

Es entonces en este contexto donde pueden existir vías de interacción, "anónimas e implícitas", que hacen posible la circulación del conocimiento e información dentro de las organizaciones. En términos de una red de investigadores estas fuentes relacionales entregan, en menor o mayor medida, atributos de poder e influencia a 
REDES- Revista hispana para el análisis de redes sociales

Vol.14,\#5, J unio 2008

http://revista-redes.rediris.es

algunos investigadores (generando posiciones diferenciales), ya que pasan a ser facilitadores (o no) y/o transportadores (o no) de información y conocimiento privilegiado. En consecuencia, nuestro objetivo apunta a identificar grupos, o individuos, cuyo poder e influencia se funda en atributos relacionales más que formales -cargos profesionales-, ampliando la visión sobre el desarrollo y la circulación del conocimiento organizativo, tomando en cuenta la existencia de redes de interacción, con preponderancia en lo que se ha conceptualizado como capital social[7].

Dentro de la diversidad de perspectivas que asocian la teoría del capital social con el análisis de redes es importante considerar la propuesta de Ronald Burt (2000), en donde responde, y se opone, a la argumentación de un capital social definido exclusivamente a partir de la cohesión de los grupos en cuanto lazos de confianza y reciprocidad, y con énfasis en la densidad de las redes[8]. Es decir, desecha la importancia de la densidad de las redes para enfocarse más en la calidad de las mismas, midiéndose ésta en función de la posibilidad de acceso a información referente a entornos lejanos e inaccesibles al individuo por sí solo (structural holes). No obstante Burt concuerda con Coleman, y por ende con Putnam, en que ciertas estructuras sociales son capaces de crear para ciertos individuos o grupos ventajas competitivas a la hora de alcanzar sus propios intereses, y en que los individuos mejor conectados son quienes disfrutan de mayores beneficios. El desacuerdo aparece a la hora de definir qué significa estar "mejor conectado". Burt describe el capital social por su función de mediación en cuanto al acceso de oportunidades, y denomina conexiones "de puente" a las que logran conectar a los individuos con grupos distintos al de pertenencia. Así, las conexiones que tienden puentes hacia otros grupos constituyen una ventaja respecto de las posibilidades de acceso a información, ya que estos individuos alcanzan un volumen mayor de información al relacionarse indirectamente con un mayor número de personas, pero más importante aún, es la diversidad de los contactos la que garantiza, de algún modo, que esa información no será redundante. Estos individuos también cuentan con una ventaja de control, esto es, con la posibilidad de poner en contacto ciertas personas en pos de algún interés personal. "De este modo, individuos en contacto con redes ricas en agujeros estructurales[9] son los que saben acerca de y ejercitan control sobre. Por lo tanto, la construcción de capital social no parte sólo del establecimiento de vínculos estrechos, sino también de la capacidad de los actores para establecer diferentes relaciones fuera de su grupo de pertenencia.

Esta visión del capital social permitirá obtener una perspectiva adicional y complementaria de la influencia o poder dentro de nuestro estudio de caso, más 
REDES- Revista hispana para el análisis de redes sociales

Vol.14,\#5, J unio 2008

http: // revista-redes.rediris.es

específicamente, dentro de la red de investigación que pueda conformarse entre los investigadores del departamento, identificando aquellos individuos "puente", y que con ello, los que detentan un poder relacional distinto al que pueden entregar los atributos formales (cargos), aún más, ante la eventualidad de que se concreten conexiones con investigadores externos al departamento.

En función del interés de este trabajo se definirán tres dimensiones del capital social como determinantes en la creación y producción de conocimiento mediante redes sociales (Julve, 2000), pertinentes a tener en cuenta al momento de identificar la importancia de los agujeros estructurales:

1. Conectiva: Indica los recursos que se encuentran al alcance de individuo, la posición que el individuo tenga en la red será primordial a la hora de poder acceder a los recursos que circulan por la red. Determinará las oportunidades que pueda tener para acceder a nuevos conocimientos, por consiguiente, habrán individuos que tengan más ventajas que otros en términos de exclusividad, acceso y tiempo.

Estas oportunidades y diferenciaciones de los individuos en la red serán determinadas por lo que hemos denominado como huecos estructurales, definidos como la ausencia de conexiones entre ciertos individuos en ciertos sectores de la red, aportando también grandes beneficios informativos para aquellos nodos que sean puente o conecten sectores de la red con ausencia de relaciones.

2. Coordinadora: Facilita la integración, acceso y selección de los recursos que se encuentran en la red. Esta integración va determinada por las conexiones que los individuos tengan en la red, independientemente de las tareas que estos realicen en la organización. Determinados tipos de estructura social facilitan la generación de obligaciones y expectativas mutuas, normas y convenciones sociales, y esquemas compartidos de interpretación, que incrementan la previsibilidad y confianza en el comportamiento de los actores interdependientes, y contribuyen a disminuir los costes de coordinación.

3. Motivacional: Proporciona la compensación para los individuos que se implican en el proceso y en la red. De esta forma por ejemplo, el hecho de pertenecer a una cierta red puede dar cierta estima a los individuos que la componen, favoreciendo su satisfacción a la hora de realizar sus labores. Es decir, genera una compensación a través de los créditos sociales que 
REDES- Revista hispana para el análisis de redes sociales

Vol.14,\#5, J unio 2008

http://revista-redes.rediris.es

obtienen los individuos por las acciones desinteresadas (favores) que puedan hacer a otros miembros de la red.

En resumen, la primera dimensión está determinada por la posición que tenga cada individuo en la red, por otro lado, las dimensiones coordinadora y motivacional devienen de la conectividad y morfología de la red en la cual se relacionan los individuos.

\section{Estudio de caso: Las relaciones de investigadores en el departamento de académicos}

Para esta investigación se seleccionó a todos aquellos investigadores que tuvieron alguna relación contractual o de colaboración con el departamento académico, entendiendo esta relación como la participación formal en algún proyecto de investigación, contrato $\mathrm{y} / \mathrm{o}$ convenio de investigación durante el periodo comprendido entre Febrero -2004 y Junio -2006. [10]. Para los cálculos realizados para el análisis se utilizaron los Software UCINET 6 y SPSS.

\section{A. Exploración de la red}

Como primer paso para la exploración, se presenta en la llustración 1 una imagen de la red de investigadores del departamento académico. La imagen permite visualizar a grandes rasgos algunas propiedades generales de la red de investigadores, donde cada investigador está representado por un nodo, y cada unión es la coincidencia de los investigadores en al menos uno de los proyectos de investigación. 
REDES- Revista hispana para el análisis de redes sociales

Vol.14,\#5, J unio 2008

http: // revista-redes.rediris.es

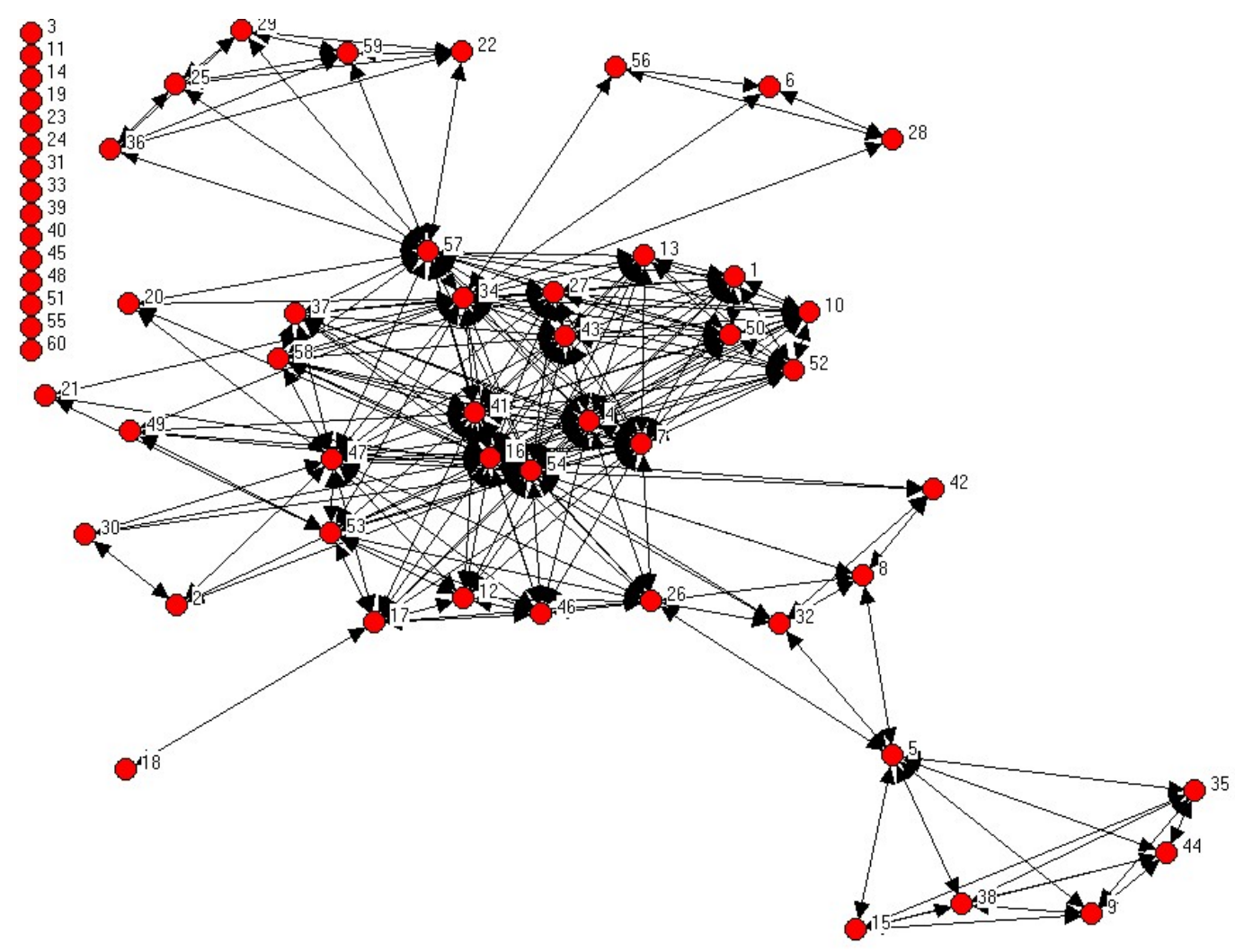

I lustración 1. Red de Investigadores.

Tal como se puede apreciar en la figura 1, la red de investigadores a través de los distintos proyectos muestra ciertas particularidades y características relevantes, tales como:

- NO existe una red totalmente estructurada, el $25 \%$ del total del personal nunca han participado en un proyecto de investigación con otros investigadores.

- Una segunda característica es la alta densidad que se puede observar en el centro de la red, lo que podría indicar la existencia de un grupo de investigación, más o menos estable, y probablemente, conformado por la elite académica del departamento.

- En la periferia de la red, pueden identificarse ciertos grupos de investigadores que podrían conformar, por un lado, otros grupos de investigación, o bien, investigadores periféricos al departamento que acceden a la red por algún nodo intermediador específico. En cualquiera de estos casos, lo que si está claro, es que su participación en la red se encuentra altamente intermediada, y en consecuencia, existe una limitada participación para acceder a los recursos que pueden circular por la red. 
REDES- Revista hispana para el análisis de redes sociales

Vol.14,\#5, J unio 2008

http://revista-redes.rediris.es

\section{A.1. Tamaño y Distancia de la red}

El tamaño de una red es muy importante, ya que determina la estructura de las relaciones, debido a que cada actor dispone de recursos limitados para crear y mantener relaciones. De esta forma, una red extensa conlleva que los nodos realicen un gran esfuerzo para poder participar con otros nodos de la red. Para el caso aquí analizado, se puede definir una red de tamaño considerable que alcanza los 60 nodos. En este contexto, la distancia puede ser un buen indicador del tamaño de la red:

\section{Average distance (among reachable pairs) $=\mathbf{2 . 2 8 6}$}

Lo anterior, indica que los actores o nodos, en promedio, se ven intermediados por al menos dos nodos para relacionarse con el resto de nodos en la red, confirmando la considerable extensión de la red.

\section{Distance-based cohesion $=\mathbf{0} \mathbf{0 . 3 0 6}$}

(range 0 to 1; larger values indicate greater cohesiveness)

Por otro lado, la distancia basada en la cohesión para esta red alcanza un valor de 0.3 , que en una escala de 0 a 1 , puede ser considerada como baja, esta condición se debe en gran parte a la existencia una cantidad considerable de nodos desconectados totalmente de la red, distorsionando así la tasa de distancia presente en la red principal.

En consecuencia, estos datos en su conjunto dan cuenta del gran esfuerzo que los investigadores (en promedio) tendrán que realizar para acceder a los distintos recursos que circulan por la red, y/o que se puedan producir en los distintos grupos o áreas de la red.

\section{A.2. Densidad de la Red}

El grado de densidad es otro indicador importante para definir la estructura de relaciones en una red, ya que cuantifica la cantidad promedio de lazos que se crean dentro de la red, y para este caso se obtuvo una densidad de:

Density (matrix average) $=\mathbf{0 . 1 7 2 9}$

Standard deviation $\mathbf{= 0 . 5 1 8 1}$ 
REDES- Revista hispana para el análisis de redes sociales

Vol.14,\#5, J unio 2008

http: // revista-redes.rediris.es

El valor obtenido puede ser considerado como bastante "bajo", y viene a confirmar la idea de la escasa conectividad de la red, ya que sólo se alcanza un 17,3\% respecto al potencial de conexión entre los actores. Sin embrago, esta baja conectividad no es una propiedad uniforme de toda la red. Tal como se observa en la figura 1 existen ciertas zonas con una conectividad altísima, esto último se ve confirmado con la desviación estándar de la densidad (53 \%), lo que en definitiva, se viene a traducir en una importante centralidad de algunos nodos, y por el contrario, la nula conexión de otros, en consecuencia, una marcada desigualdad para acceder o introducir información y conocimientos en la red.

\section{A.3. Centralidad de la Red}

De acuerdo a los datos la Tabla 1, en promedio, los actores tienen 10,2 grados de centralidad en la red, algo bastante bajo si se considera el tamaño de la red. Por otro lado, si se observan los valores máximos y mínimos, vemos que existe al menos un nodo con 51 grados y otro con 0 grado, lo que puede indicar que el promedio se debe a que existe una cantidad considerable de nodos con valores extremos. Esto último se ve claramente reafirmado por el coeficiente de variación ([Std Dev/ Mean]*100) que alcanza un valor de 124,5, considerado bastante alto, confirmando que en esta red las ventajas posicionales se distribuyen muy desigualmente entre los nodos.

$\begin{array}{rrrrr} & & 1 & 2 & 3 \\ & & \text { Degree } & \text { NrmDegree } & \text { Share } \\ 1 & \text { Mean } & 10.200 & 2.881 & 0.017 \\ 2 & \text { Std Dev } & 12.737 & 3.598 & 0.021 \\ 3 & \text { Sum } & 612.000 & 172.881 & 1.000 \\ 4 & \text { Variance } & 162.227 & 12.945 & 0.000 \\ 5 & \text { SSQ } & 15976.000 & 1274.857 & 0.043 \\ 6 & \text { MCSSQ } & 9733.600 & 776.724 & 0.026 \\ 7 & \text { Euc Norm } & 126.396 & 35.705 & 0.207 \\ 8 & \text { Minimum } & 0.000 & 0.000 & 0.000 \\ 9 & \text { Maximum } & 51.000 & 14.407 & 0.083\end{array}$

Tabla 1. Centralidad de la red

\section{B. Centralidad de grado}

La idea principal de este apartado es identificar y analizar fehacientemente la relación que pueda existir entre el cargo (o categoría profesional) y el grado de centralidad en la red.

Como primera aproximación, se presenta la red de investigadores caracterizada en términos de la centralidad de grado de los nodos (tamaño) y del cargo que desempeñan en el departamento (color). 
REDES- Revista hispana para el análisis de redes sociales

Vol.14,\#5, J unio 2008

http: // revista-redes.rediris.es
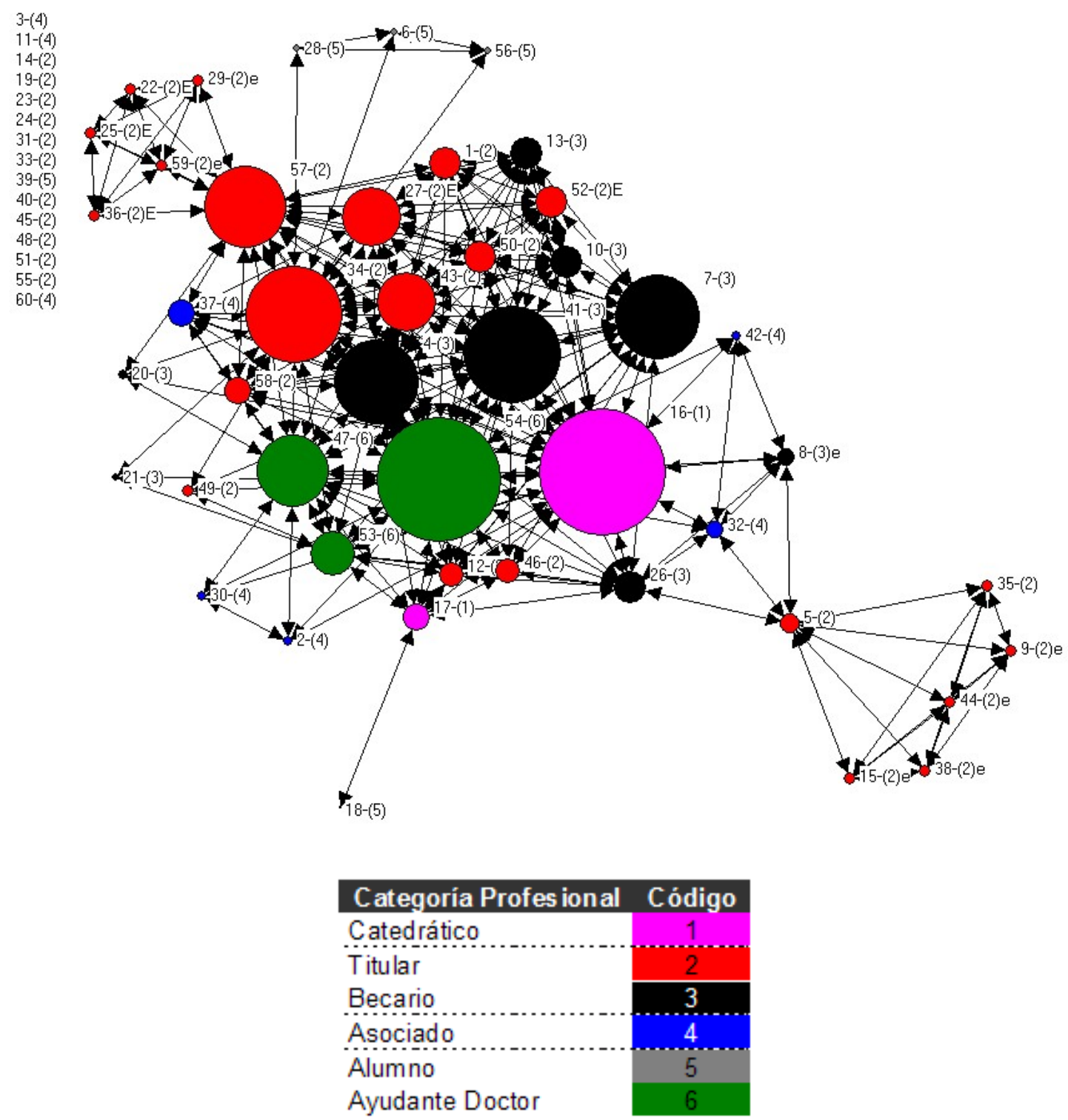

I lustración 2. Red de investigadores caracterizada por categoría profesional y centralidad

Tal como se puede apreciar (Ilustración 2), no pareciera existir una relación clara entre el cargo desempeñado dentro del departamento con un posición más o menos central dentro de la red de investigadores. A tal punto, y a modo de ejemplo, se puede apreciar a becarios y/o ayudantes con centralidades mayores que profesores titulares. Así también, podemos observar cómo los nodos aislados de la red, en su mayoría, son profesores titulares, un cargo académico efectivamente más relevante que un alumno, becario o asociado.

Lo que si pareciera indicar esta imagen es que los nodos cuyos cargos son asociado y alumno tendrían una menor centralidad dentro de la red (azul y plomo), quizás debido a su relación más esporádica en términos académicos y contractuales con el departamento. No obstante lo anterior, pereciera cumplirse la afirmación de que el 
REDES- Revista hispana para el análisis de redes sociales

Vol.14,\#5, Junio 2008

http://revista-redes.rediris.es

cargo no se correlacionaría con una posición determinada en la red de investigadores. Para corroborar esta afirmación, realizaremos un análisis estadístico entre el cargo desempeñado y la centralidad obtenida.

\section{B.1 Análisis estadístico}

En una primera aproximación a este análisis se generó un reporte de la centralidad media para cada tipo de cargo, tal como se puede ver en la siguiente tabla[11].

Centralidad por Grado
\begin{tabular}{|l|r|r|r|}
\hline Cat. Profesional & \multicolumn{1}{|c|}{ Media } & $\mathrm{N}$ & Desv. típ. \\
\hline Catedrático & 31,0000 & 2 & 28,28427 \\
Titular & 7,6667 & 33 & 9,66523 \\
Becario & 17,7778 & 9 & 14,00694 \\
Asociado & 3,7500 & 8 & 3,88219 \\
Alumno & 2,0000 & 5 & 1,41421 \\
AyDoctor & 32,3333 & 3 & 16,25833 \\
Total & 10,2000 & 60 & 12,84431 \\
\hline
\end{tabular}

Tabla 2. Centralidad media por cada categoría profesional

El test ANOVA, con categorías profesionales re-codificadas, nos permite observar si existe una diferencia significativa de las medias de centralidad en función de los cargos desempeñados por los investigadores.

Recodificación de los cargos:

a) Profesor $=$ Catedrático + Titular + Ayudante Doctor

b) Becario = Becario + Alumno

C) Asociado.

Tabla de ANOVA

\begin{tabular}{|c|c|c|c|c|c|c|c|}
\hline & & & $\begin{array}{l}\text { Suma de } \\
\text { cuadrados }\end{array}$ & gl & $\begin{array}{c}\text { Media } \\
\text { cuadrática }\end{array}$ & $\mathrm{F}$ & Sig. \\
\hline \multirow{5}{*}{$\begin{array}{l}\text { Centralidad por Grado } \\
\text { * Cargo Profesores } \\
\text { Agrupados }\end{array}$} & Inter-grupos & (Combinadas) & 401,333 & 2 & 200,667 & 1,226 & ,301 \\
\hline & & Linealidad & 186,323 & 1 & 186,323 & 1,138 & 291 \\
\hline & & $\begin{array}{l}\text { Desviación de } \\
\text { la linealidad }\end{array}$ & 215,011 & 1 & 215,011 & 1,313 & 257 \\
\hline & Intra-grupos & & 9332,267 & 57 & 163,724 & & \\
\hline & Total & & 9733,600 & 59 & & & \\
\hline
\end{tabular}

Tabla 3. Test ANOVA para centralidad y categorías profesionales re-codificadas (1) 
REDES- Revista hispana para el análisis de redes sociales

Vol.14,\#5, J unio 2008

http: // revista-redes.rediris.es

A simple vista se puede concluir que la prueba ANOVA no muestra indicadores significativos para rechazar la hipótesis nula, entendiendo esta como "no existe variabilidad significativa de la varianza de la centralidad entre las distintas categorías profesionales[12]".

Tomando en consideración que la categoría Asociado sólo toma 7 grados de libertad, se procedió a realizar una nueva recodificación, esta vez, dejando la variable cargo con dos categorías, -sumando la categoría becario a la de asociado-. En este contexto, se aplicó una prueba t para establecer diferencia de medias entre muestras independientes.

a) Grupo 1: Profesor (Catedrático + Titular + Ayudante Doctor)

b) Grupo 2: Asociado-Becario (Becario + Alumno)

Estadísticos de grupo

\begin{tabular}{|c|c|c|c|c|c|}
\hline & $\begin{array}{l}\text { Profesor_- } \\
\text { AsociadoBecario }\end{array}$ & $\mathrm{N}$ & Media & $\begin{array}{l}\text { Desviación } \\
\text { típ. }\end{array}$ & $\begin{array}{c}\text { Error típ. de } \\
\text { la media }\end{array}$ \\
\hline \multirow[t]{2}{*}{ Centralidad por Grado } & Profesor & 38 & 10,8421 & 13,60550 & 2,20710 \\
\hline & Asociado_Becario & 22 & 9,0909 & 11,63291 & 2,48015 \\
\hline
\end{tabular}

Tabla 4. Test de diferencias de medias para centralidad y categoría profesionales recodificadas (2)

Prueba de muestras independientes

\begin{tabular}{|c|c|c|c|c|c|c|c|c|c|c|}
\hline & & \multicolumn{2}{|c|}{$\begin{array}{c}\text { Prueba de Levene } \\
\text { para la igualdad de } \\
\text { varianzas }\end{array}$} & \multicolumn{7}{|c|}{ Prueba T para la igualdad de medias } \\
\hline & & \multirow[b]{2}{*}{$\mathrm{F}$} & \multirow[b]{2}{*}{ Sig. } & \multirow[b]{2}{*}{$\mathrm{t}$} & \multirow[b]{2}{*}{$\mathrm{gl}$} & \multirow[b]{2}{*}{ Sig. (bilateral) } & \multirow{2}{*}{$\begin{array}{l}\text { Diferencia } \\
\text { de medias }\end{array}$} & \multirow{2}{*}{$\begin{array}{l}\text { Error típ. de } \\
\text { la diferencia }\end{array}$} & \multicolumn{2}{|c|}{$\begin{array}{c}95 \% \text { Intervalo de } \\
\text { confianza para la } \\
\text { diferencia }\end{array}$} \\
\hline & & & & & & & & & Inferior & Superior \\
\hline Centralidad & $\begin{array}{l}\text { Se han asumido } \\
\text { varianzas iguales }\end{array}$ & ,292 &, 591 &,- 372 & 58 & ,712 & $-1,29665$ & 3,48936 & $-8,28135$ & 5,68805 \\
\hline & $\begin{array}{l}\text { No se han asumido } \\
\text { varianzas iguales }\end{array}$ & & &,- 379 & 46,818 & ,706 & $-1,29665$ & 3,41747 & $-8,17243$ & 5,57912 \\
\hline
\end{tabular}

Tabla 5. Prueba $t$ de diferencia de medias a partir de centralidad de grado

Tal como se pude ver en la tabla 5, tampoco existiría una diferencia de medias significativa entre ambos grupos, concluyendo que no existe evidencia suficiente para rechazar la hipótesis nula (Ho: no existe diferencia de medias entre ambos grupos) con un $95 \%$ de confiabilidad. Por lo tanto, nuevamente no se puede establecer una relación significativa entre ambas variables. Así, a la luz de los datos, podría esperarse que el cargo profesional no sea necesariamente una variable determinante a la hora de ocupar una posición y/o detentar ciertos atributos relacionales dentro de la red, y por ende, la apropiación de los recursos que por esta circulen. 
REDES- Revista hispana para el análisis de redes sociales

Vol.14,\#5, J unio 2008

http: // revista-redes.rediris.es

\section{Centralidad por intermediación}

Este tipo de centralidad define el poder que tienen ciertos nodos dentro de una red para conectar o ser "puente" entre ciertos grupos de nodos, y en consecuencia, se identifican como nodos "bisagras" en el manejo de los recursos que puedan fluir por la red.

En la tabla siguiente se describen los principales indicadores de intermediación para la red de investigadores.

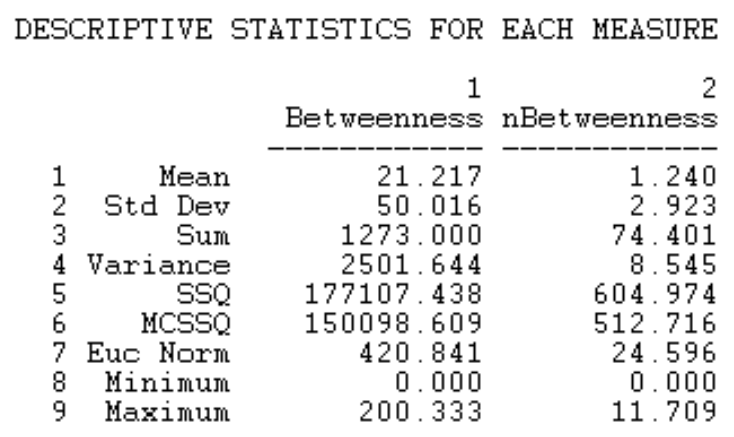

Tabla 6. Indicadores de intermediación global

En términos generales, nuevamente se observa una desigual distribución de los atributos de intermediación en la red, ya que existe una gran variación en las puntuaciones para los distintos actores, con nodos que no alcanzan valores mayores que cero, y por el contrario, otros que alcanzan puntuaciones máximas de 200 puntos. Esto último se ve reafirmado al visualizar una media muy baja de 11,067 puntos, determinada por una alta desviación estándar ( $50.01 \%$ ). Por tanto, y tal como se visualizaba en los análisis exploratorios anteriores, cabe esperar que en esta red existan muy pocos nodos con altos puntajes de intermediación. En la siguiente tabla se da cuenta de los nodos que alcanzan los mayores valores de intermediación.

\begin{tabular}{|c|c|c|}
\hline & Intermediación & \\
\hline & ID + cargo & Valor \\
\hline 1 & 57-(profesor) & 200,3 \\
\hline 2 & 5-(Profesor) & 195,0 \\
\hline 3 & 54-(Ayudante doctor) & 154,2 \\
\hline 4 & 16-(Catedrático) & 148,2 \\
\hline 5 & 34-(profesor) & 146,3 \\
\hline 6 & 26-(Becario) & 146,1 \\
\hline 7 & 47-(Ayudante doctor) & 45,2 \\
\hline 8 & 17-(Catedrático) & 43,0 \\
\hline 9 & 8-(Becario) & 38,2 \\
\hline 10 & 32-(Asociado) & 38, \\
\hline 11 & 41-(Becario) & 38,0 \\
\hline
\end{tabular}


REDES- Revista hispana para el análisis de redes sociales

Vol.14,\#5, J unio 2008

http: // revista-redes.rediris.es

\begin{tabular}{|l|c|c|}
12 & 7-(Becario) & 31,6 \\
\hline 13 & 4-(Becario) & 31,6 \\
14 & 53-(Ayudante doctor) & 13,6 \\
15 & 43-(profesor) & 1,7 \\
16 & 27-(profesor) & 1,7 \\
\hline
\end{tabular}

Tabla 6. Indicadores de intermediación específicos de los nodos

En el análisis de este indicador se puede observar que sólo 16 actores (27\% del total) tienen valores mayores a 0 , es decir, tienen el "poder" de comunicar a un grupo de nodos con el resto de la red. En el contexto de esta investigación se puede definir a estos actores como nodos conectores entre participantes de distintos proyectos de investigación, y a su vez, intermediarios de los conocimientos que se generan en los mismos. Es necesario considerar que sólo intermedian los caminos más cortos (geodésicos), y no caminos alternativos, si bien estos últimos no son los más eficientes, si son una alternativa válida a la hora de buscar integración dentro de la red.

Con excepción del nodo no 5, dentro de los primeros lugares de esta clasificación se vuelven a posicionar los mismos nodos que alcanzan altos valores de centralidad por grado. En consecuencia, son nodos que están muy bien conectados en su vecindario, pueden influir fácilmente al resto de nodos, y además, intermedian y/o controlan información de cierto(s) grupo(s) de nodos con el resto de la red. 
REDES- Revista hispana para el análisis de redes sociales

Vol.14,\#5, J unio 2008

http: // revista-redes.rediris.es

Una característica especial de los nodos "puente" 5 y 57 es que se relacionan con redes externas al departamento, lo cual implica que además de intermediar la información que fluya desde interior de la red, también intermedia la información proveniente de redes externas, muy importantes por ser novedosas y poco redundantes. Esta última característica implica un alto poder y control de la información que sale y entra en la red. En la figura siguiente se ejemplifica la relación de intermediación de los nodos citados con nodos externos (redes externas).
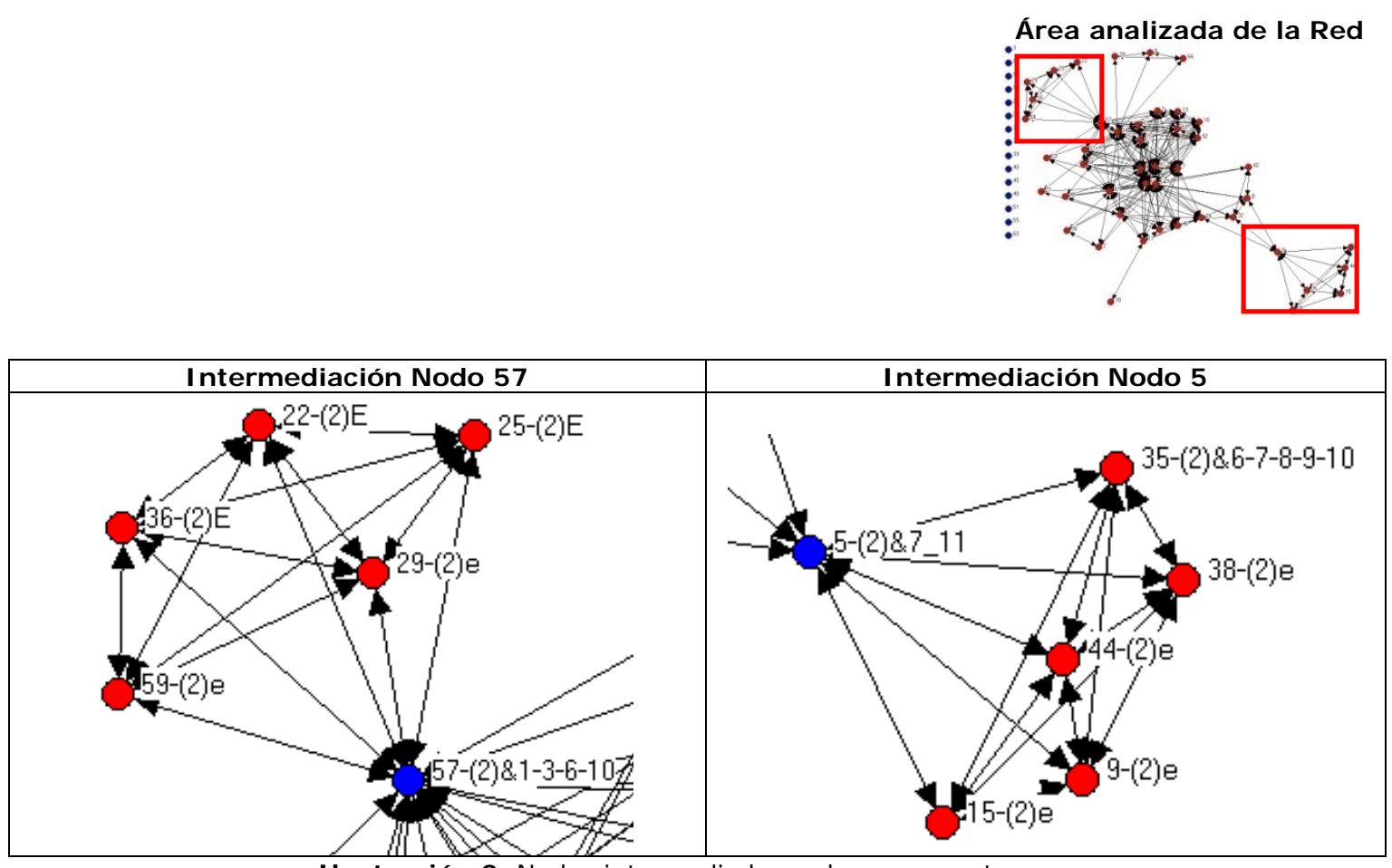

I lustración 3. Nodos intermediadores de grupos externos.

Nota: El primer número de un nodo es su identificador, el número entre paréntesis indica la categoría profesional dentro del departamento, y el tercer número indica el área de interés del investigador. Cuando es un letra " $\mathrm{e}$ " se asume que es un investigador externo al departamento estudiado.

Otro punto a considerar es la intermediación que realiza el nodo 34 , ya que el grupo de alumnos que "conecta" es cualitativamente distinto de los grupos comentados anteriormente. Al tratarse de estudiantes o alumnos, se definen más como un grupo "receptor" de información y conocimiento que generadores del mismo. En este contexto, la función del nodo 34 será más bien la de "entregar" información a este grupo, y en menor grado, la de "recoger" recursos. Por consiguiente, el poder relacional de este nodo será mucho menor que el de los nodos 5 y 57 . 
REDES- Revista hispana para el análisis de redes sociales

Vol.14,\#5, J unio 2008

http: // revista-redes.rediris.es

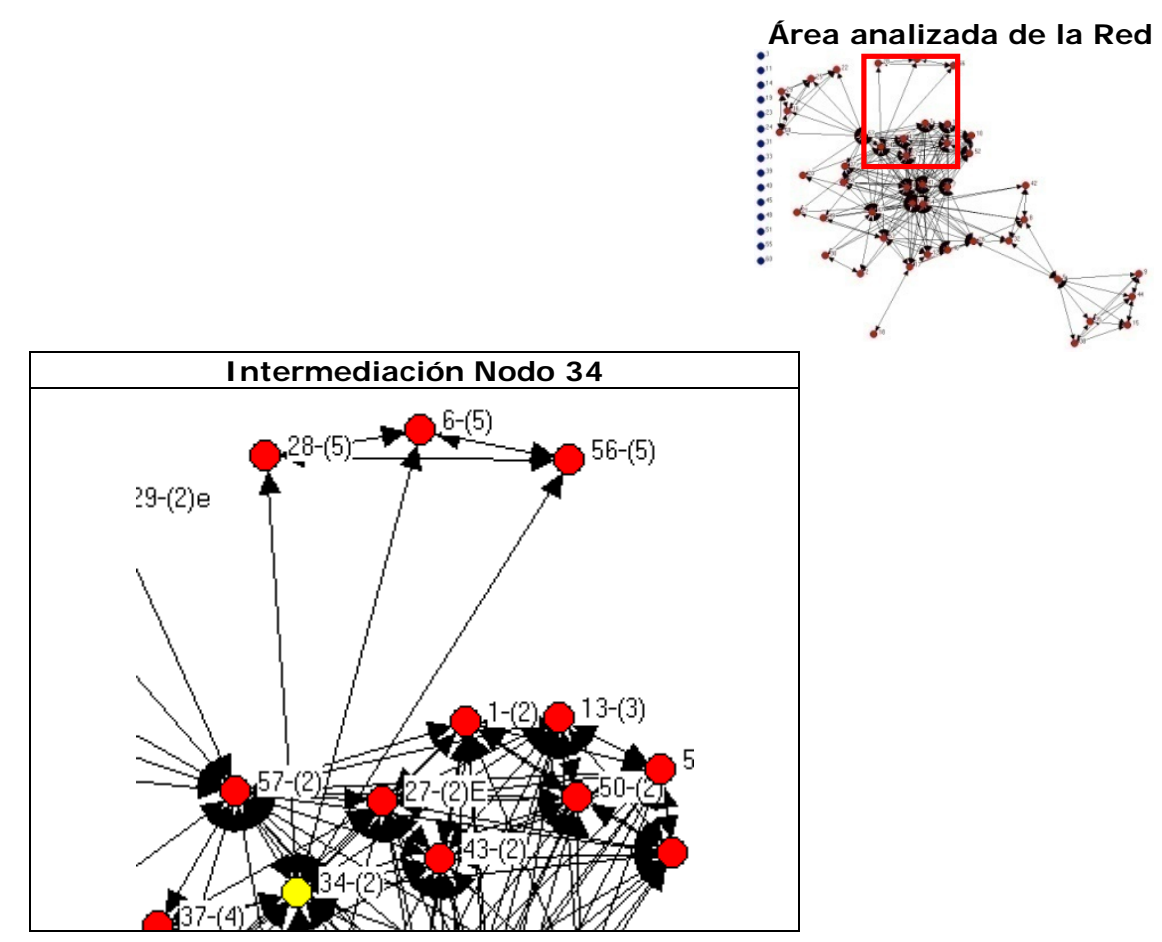

I lustración 4. Nodo intermediador de un grupo de alumnos.

\section{C.1 Análisis estadístico}

El análisis de estos datos muestra y confirma la dispersión desigual de la centralidad por intermediación entre los distintos tipos de investigadores. A tal punto, que la categoría alumno no alcanza siquiera niveles de intermediación cuantificables.

Centralidad por Intermediación
\begin{tabular}{|l|r|r|r|}
\hline Cat. Profesional & \multicolumn{1}{|c|}{ Media } & N & Desv. típ. \\
\hline Catedrático & 102,3645 & 2 & 83,95408 \\
Titular & 16,3999 & 33 & 52,91586 \\
Becario & 31,7032 & 9 & 46,21927 \\
Asociado & 4,8029 & 8 & 13,58458 \\
Alumno &, 0000 & 5 &, 00000 \\
AyDoctor & 67,4413 & 3 & 69,86992 \\
Total & 21,2000 & 60 & 50,51225 \\
\hline
\end{tabular}

Tabla 7. Centralidad de intermediación media por cada categoría profesional descriptivos. 
REDES- Revista hispana para el análisis de redes sociales

Vol.14,\#5, J unio 2008

http: // revista-redes.rediris.es

La prueba de ANOVA realizada para las distintas categorías profesionales tampoco arroja significación para establecer diferencias de varianza de centralidad significativas en cada una de las categorías mencionadas[13].

Tabla de ANOVA

\begin{tabular}{|c|c|c|c|c|c|c|c|}
\hline & & & $\begin{array}{l}\text { Suma de } \\
\text { cuadrados }\end{array}$ & gl & $\begin{array}{c}\text { Media } \\
\text { cuadrática }\end{array}$ & $\mathrm{F}$ & Sig. \\
\hline \multirow{5}{*}{$\begin{array}{l}\text { Centralidad por } \\
\text { Intermediación * Cargo } \\
\text { Profesores Agrupados }\end{array}$} & Inter-grupos & (Combinadas) & 2695,818 & $\overline{2}$ & 1347,909 &, 520 &, 598 \\
\hline & & Linealidad & 2418,723 & 1 & 2418,723 & ,933 & ,338 \\
\hline & & $\begin{array}{l}\text { Desviación de } \\
\text { la linealidad }\end{array}$ & 277,095 & 1 & 277,095 & 107 & ,745 \\
\hline & Intra-grupos & & 147841,951 & 57 & 2593,718 & & \\
\hline & Total & & 150537,768 & 59 & & & \\
\hline
\end{tabular}

Tabla 8. Test ANOVA para centralidad de intermediación y categorías profesionales re-codificadas (1).

Realizando el mismo ejercicio que en el punto anterior, para la medición de la centralidad de grado, se re-codificaron nuevamente las categorías profesionales[14], y se aplicó la denominada prueba T para diferencia de medias.

Prueba de muestras independientes

\begin{tabular}{|c|c|c|c|c|c|c|c|c|c|}
\hline & \multicolumn{2}{|c|}{$\begin{array}{c}\text { Prueba de Levene } \\
\text { para la igualdad de } \\
\text { varianzas }\end{array}$} & \multicolumn{7}{|c|}{ Prueba T para la igualdad de medias } \\
\hline & \multirow[b]{2}{*}{$\mathrm{F}$} & \multirow[b]{2}{*}{ Sig. } & \multirow[b]{2}{*}{$\mathrm{t}$} & \multirow[b]{2}{*}{$\mathrm{gl}$} & \multirow[b]{2}{*}{ Sig. (bilateral) } & \multirow{2}{*}{$\begin{array}{l}\text { Diferencia } \\
\text { de medias }\end{array}$} & \multirow{2}{*}{$\begin{array}{l}\text { Error típ. de } \\
\text { la diferencia }\end{array}$} & \multicolumn{2}{|c|}{$\begin{array}{c}\text { 95\% Intervalo de } \\
\text { confianza para la } \\
\text { diferencia }\end{array}$} \\
\hline & & & & & & & & Inferior & Superior \\
\hline $\begin{array}{l}\text { Centralidad por Se han asumido } \\
\text { Intermediación varianzas iguales }\end{array}$ & 3,541 & ,065 &, 754 & 58 & ,454 & 10,23792 & 13,58202 & 16,94944 & 37,42528 \\
\hline
\end{tabular}

Tabla 9. Prueba t de diferencia de medias a partir de centralidad de intermediación.

Tal como se puede observar en el cuadro 8, tampoco se puede afirmar que exista una diferencia de medias que resulte significativa para ambos tipos de categorías, con un nivel de significación menor al 0,05. En consecuencia, cabe esperar que la variable "categoría profesional" no resulte ser determinante a la hora de definir la posición de intermediación de un investigador en la red.

\section{Grupos y Subgrupos}

La metodología de grupos se utiliza para identificar posiciones similares de actores en la red, de acuerdo a la relación que establecen con los otros actores circundantes. Los grupos son indicadores de centros neurálgicos, ya que son las estructuras más fuertemente cohesionadas, poniendo de manifiesto espacios de alta identificación y solidaridad entre los actores, lo que en términos de esta investigación sería identificar grupos formados por investigadores que tienen una alta frecuencia de encuentros y participación en los distintos proyectos. Es decir, unidades muy cohesionadas y transversales a los distintos proyectos. Además de 
REDES- Revista hispana para el análisis de redes sociales

Vol.14,\#5, J unio 2008

http: // revista-redes.rediris.es

identificar los grupos para corroborar los resultados obtenidos anteriormente, también se busca ver si su conformación tiene alguna relación con la similitud de áreas de conocimiento que los investigadores definen desarrollar o investigar, esto es, dar cuenta como los investigadores se reúnen en función de compartir áreas de conocimiento similares.

\section{D.1 F-Groups}

Está primera técnica, si bien es muy estricta en determinar los grupos, busca definir conjuntos de nodos al interior de la red en los cuales sus miembros tengan una alta cohesión interna (donde todos se contactan con todos), y una menor conectividad con el entorno. Y tal como venía definiéndose en apartados anteriores, existirían ciertos indicios de la existencia de un grupo de nodos que compartirían lazos "fuertes" entre ellos (enlaces con un peso igual a 3), y que además obtendrían puntajes muy altos en los distintos indicadores de centralidad y poder.

Group 4:

$\begin{array}{lllllllll}4 & 7 & 16 & 34 & 41 & 47 & 53 & 54 & 57\end{array}$

Strong ties have value 3.00 (level 3 )

Este resultado viene a confirmar la existencia de un "grupo de investigación principal" dentro de la llamada "red principal", y que sin lugar a dudas, conforma la base investigadora para el desarrollo de los distintos proyectos de investigación. A continuación, se presenta un análisis de las distintas áreas de conocimiento que estos investigadores definen como sus prioritarias, y con esto, ver el grado de coincidencia de las preferencias al interior del grupo.

\begin{tabular}{|c|c|c|c|c|c|c|c|c|c|c|c|c|}
\hline Nodos Áreas & 1 & 2 & 3 & 4 & 5 & 6 & 7 & 8 & 9 & 10 & 11 & 0 \\
\hline 16-(1) & & & & & & 6 & 7 & & 9 & 10 & & \\
\hline 54-(6) & & & & & & & 7 & & 9 & 10 & & 97 \\
\hline 34-(2) & & & 3 & & & & 7 & & 9 & 10 & & \\
\hline 41-(3) & & & & & & 6 & 7 & & & & 11 & \\
\hline 7-(3) & 1 & 2 & 3 & 4 & 5 & 6 & 7 & 8 & 9 & 10 & 11 & \\
\hline 4-(3) & 1 & & 3 & & & & 7 & & 9 & 10 & & \\
\hline 57-(2) & 1 & & 3 & & & 6 & & & & 10 & & \\
\hline 47-(6) & & & & & 5 & 6 & 7 & & 9 & 10 & & 98 \\
\hline 53-(6) & 1 & 2 & 3 & & 5 & & & & 9 & 10 & & 98 \\
\hline $\begin{array}{l}\text { Porcentaje } \\
\text { Concordancia }\end{array}$ & $44 \%$ & $22 \%$ & $56 \%$ & $11 \%$ & $33 \%$ & $56 \%$ & $78 \%$ & $11 \%$ & $78 \%$ & $89 \%$ & $22 \%$ & $33 \%$ \\
\hline
\end{tabular}

Tabla 10. Áreas de cocimiento preferidas por los investigadores del grupo. 
REDES- Revista hispana para el análisis de redes sociales

Vol.14,\#5, J unio 2008

http: // revista-redes.rediris.es

De acuerdo a la tabla anterior, se puede observar que si bien existe una heterogeneidad de elección de áreas de conocimiento por parte de los investigadores, es claro que existen tres áreas donde concuerdan claramente las preferencias de la mayoría, estas son: 7, 9 y 10, y en lugares más secundarios las áreas 3 y 6 . En consecuencia, podría inferirse que existen ciertas áreas de conocimiento que reúnen a estos investigadores para desarrollarlas. Sin embargo, al observar los cargos de cada uno de los investigadores de este grupo se ve claramente que existen nodos que no tienen un poder decisional significativo para definir las áreas de investigación a ser desarrolladas, como lo son los tres becarios y los tres ayudantes doctor que componen el grupo, más bien, estos nodos se verán "acoplados" a las áreas de conocimiento que explota el investigador principal (catedrático). En consecuencia, vemos cómo el catedrático y profesor titular (quizás en menor grado), acoplan o vinculan a miembros del departamento en torno a sus áreas de interés, lo que finalmente desemboca en la conformación de un grupo con claras líneas de investigación.

La identificación de las áreas de interés de este grupo será muy relevante a la hora de compararlas con las principales líneas de investigación que son desarrolladas por los distintos proyectos de investigación, a fin de ver en qué medida este grupo define la agenda investigadora del departamento, y en consecuencia, estar en presencia de un "colegio invisible".

D.2 Cliques (Grupos con un mínimo de 3 actores)

Ciertamente el análisis anterior permitió analizar a los grupos de investigadores altamente cohesionados, pero no permite ver la existencia de posibles grupos secundarios que puedan estar desarrollando otras líneas de investigación, y que dado su carácter secundario y menos estructurado, no compartan una relación tan estricta y ponderada como el análisis de f-groups. Bien podríamos analizar sólo los proyectos, y con ello, ver si hay investigaciones llevadas a cabo por investigadores distintos a los mencionados en el grupo anterior, sin embargo, con este análisis no podríamos saber si es un hecho aislado, o si el grupo que los llevó a cabo tiene una estructura relevante y significativa en el contexto global de los distintos proyectos.

En función de lo anterior, se procederá a realizar un análisis de cliques, para por un lado, observar grupos "secundarios" con cierta estructura relacional relevante, el grado de concordancia de las áreas de interés que definen sus integrantes, y por otro, ver si las áreas principales de estos grupos se plasman como relevantes dentro de las corrientes investigadoras del departamento. Claramente se excluyen 
REDES- Revista hispana para el análisis de redes sociales

Vol.14,\#5, J unio 2008

http:// revista-redes.rediris.es

de este análisis los grupos que integren al IP 16, ya que el grupo estaría probablemente conformado en función de este nodo.

De los 14 cliques existentes, al menos en 9 de ellos tienen como integrante al nodo 16. De los 5 grupos restantes, quedan claramente definiendo aquellos grupos formados por investigadores externos al departamento (grupo 14). El grupo denominado como no 12 está formado en su gran mayoría por becarios, lo cual implica que no tienen una autonomía suficiente como para emprender proyectos de investigación.

Finalmente, se pueden definir tres grupos totalmente conectados, y que en teoría, podrían considerarse como grupos de investigación en formación, estos son los cliques: 10, 11 y 13 .

El primer clique analizado, el no 10 , no puede ser definido totalmente en términos de las áreas de investigación preferidas por sus integrantes, ya que sólo 2 de los 6 integrantes pertenecen al departamento. Sin embargo, podría inferirse que estos investigadores, al conectarse fuertemente con investigadores externos, participan en redes externas donde, por un lado, se explotan nuevas áreas de conocimiento, y por otro, acceden a recursos nuevos y novedosos en comparación con los que circulan en la red interna del departamento.

\section{GRUPO № 10}

5-(2)\&7_11

$9-(2) e$

15-(2)e

$35-(2) \& 6-7-8-9-10$

38-(2)e

44-(2)e

En cuanto al grupo 11, se observa que las áreas más importantes son la 7 y 11 . Es relevante destacar que el nodo número 5 integra este y el grupo anterior, este hecho puede dar lugar a una plataforma para la generación de nuevos grupos de investigación, ya que al excluir la participación de los nodos del grupo de investigación principal obtienen cierto grado de independencia. Además, en este grupo el nodo no 5 es el único miembro cuyo cargo le permite, en cierto sentido, proponer y ejecutar proyectos de investigación, ya que el resto de actores son becarios más un asociado. En consecuencia, existen algunos indicios estructurales que permiten inferir la conformación de nuevas redes o subredes de investigación 
REDES- Revista hispana para el análisis de redes sociales

Vol.14,\#5, J unio 2008

http: // revista-redes.rediris.es

diferentes al grupo principal, y que suman la participación de investigadores externos al departamento.

\section{GRUPO № 11}

5- $(2) \& 7 \_11$

8-(3)e

$26-(3) \& 1-2-3-5-6-7-8-9-10-11$

$32-(4) \& 1-3-6-7-9-10-11$

Por último, el grupo número 13 está conformado por un becario, dos ayudantes doctores y un titular, los que coinciden principalmente en las áreas 9 y 10, y en menor medida la 7. Es importante indicar que este grupo comparte algunos de sus miembros con el grupo principal, ya que cuenta entre sus filas uno de los nodos más centrales del grupo principal (54). De hecho, los miembros de este grupo comparten áreas muy similares a las del grupo principal (9 y 10). Esto podría implicar que podría tratarse de un grupo anexo al principal, y cuya etapa de independencia sería más temprana que la del o los cliques analizados anteriormente.

\section{GRUPO № 13}

21- (3)\&6

$34-(2) \& 3-7-9-10-101$

$53-(6) \& 1-2-3-5-9-10-98$

$54-(6) \& 7-9-10-97$

Para completar más aún más este análisis, se presenta a continuación una matriz que muestra cómo se agrupan los investigadores en torno a los 14 cliques encontrados. 
REDES- Revista hispana para el análisis de redes sociales

Vol.14,\#5, J unio 2008

http://revista-redes.rediris.es

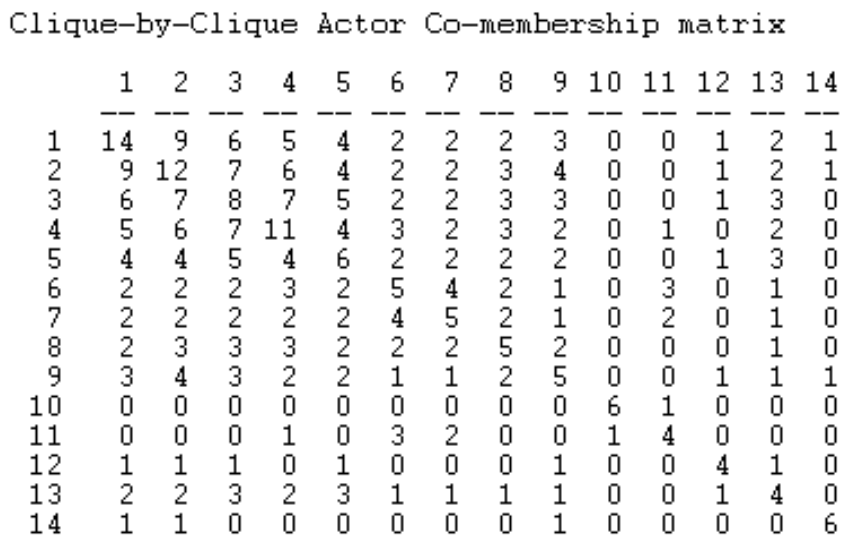

Tabla 11. Matriz de membresía de los actores a los distintos grupos.

La importancia de esta matriz radica en que permite identificar cómo los cliques agrupan a los nodos o investigadores, y de qué forma algunos de ellos se yuxtaponen o coparticipan en distintos grupos.

La diagonal de esta matriz muestra el número total de nodos que componen cada clique. Por otro lado, los valores de las columnas indican la cantidad de nodos que comparten los cliques entre sí. Ante esto, se puede observar cómo los primeros cliques, (donde la presencia de nodos del grupo de investigación principal es "de peso"), congregan y comparten a una gran cantidad de investigadores. Sin embargo, y tal como se describió en el punto anterior, vemos como los cliques 10 y 11 casi no comparten actores con los otros cliques, es más, se ve claramente cómo el clique $\mathrm{n} 010$ es totalmente cerrado a los otros grupos. Por otro lado, también vemos cómo el clique 13, algo más cercano al grupo principal, comparte una gran cantidad de nodos con los primeros cliques, confirmando su cercanía con el grupo principal.

\section{E. Principales áreas de investigación explotadas en los proyectos de investigación.}

Luego de identificar las principales áreas de investigación explotadas por los 17 proyectos de investigación analizados, se procedió a ponderar la importancia relativa de cada una de ellas en función del tiempo de duración de cada proyecto, el número de investigadores que participan y la centralidad de cada proyecto (esto último, mediante un análisis de redes de proyectos, pero que no se presenta en este documento ya que escapa al alcance de este trabajo) se obtuvo la siguiente tabla de explotación. 
REDES- Revista hispana para el análisis de redes sociales

Vol.14,\#5, J unio 2008

http: // revista-redes.rediris.es

\begin{tabular}{|cc|}
\hline $\begin{array}{c}\text { Áreas } \\
\text { Investigadas }\end{array}$ & $\begin{array}{c}\text { Porcentaje } \\
\text { de explotación }\end{array}$ \\
\hline 10 & $\mathbf{7 3 \%}$ \\
\hline 11 & $\mathbf{4 7 \%}$ \\
\hline 6 & $40 \%$ \\
\hline 7 & $38 \%$ \\
\hline 9 & $33 \%$ \\
\hline 3 & $17 \%$ \\
\hline 1 & $12 \%$ \\
\hline 2 & $8 \%$ \\
\hline 4 & $0 \%$ \\
\hline 5 & $0 \%$ \\
\hline 8 & $0 \%$ \\
\hline
\end{tabular}

Tabla 12: Listado de áreas de conocimiento y el porcentaje de explotación de cada una por la totalidad de proyectos.

Una de las primeras conclusiones que se pueden obtener es que existe una desigual explotación de las distintas áreas, unas muy desarrolladas (10 y 11 y 6), y otras sobre las cuales no se ha desarrollado investigación alguna $(4,5$ y 8$)$.

Claramente se observa cómo las áreas más explotadas se correlacionan con las áreas definidas como "preferidas" por el grupo de investigación principal en esta red, el f-group (áreas: 10, 9 y 7 - 3 y 6), en consecuencia, queda claramente establecido que este grupo de investigadores plasma sus intereses investigativos en las principales líneas de investigación que se desarrollan en el departamento. También es necesario considerar la importancia que alcanza el área no 11, una de las "preferidas" por el grupo de investigadores independientes (clique № 11), y siendo más específico, área también preferida por el nodo no 5 que actúa como IP para los grupos independientes 10 y 11 . 
REDES- Revista hispana para el análisis de redes sociales

Vol.14,\#5, J unio 2008

http://revista-redes.rediris.es

\section{Conclusiones}

El análisis de redes sociales (ARS) nos ha permitido generar una serie de indicadores replicables y comparables en la indagación de nuevas variables cuantificables en fenómenos ampliamente difundidos en el campo de desarrollo de las ciencias sociales, donde hemos podido ejemplificar, a partir de la preocupación por la generación y transmisión de conocimiento, cómo es posible describir fenómenos organizacionales de suma relevancia para la circulación del conocimiento y la información dentro de nuestro propio campo de acción.

Hemos podido dar cuenta de su versatilidad, en la medida en que es una herramienta de investigación acorde con un desarrollo intelectual precedente, como lo han sido las diversas corrientes del capital social, permitiendo hacer distinciones y diferenciaciones de grado entre actores componentes de una organización, radicando su importancia para nuestros fines en que nos ha permitido definir las siguientes conclusiones específicas:

1. La red de investigadores del departamento es bastante extensa. Primero por la cantidad de nodos que forman parte, y segundo, por la inclusión de investigadores no adscritos al departamento, lo que permite incorporar de forma más o menos directa conocimientos e información que puedan provenir de redes externas.

2. A la vez, esta red también se puede definir como incompleta, ya que al menos el $25 \%$ del personal docente e investigador (PDI) no participa en los distintos proyectos de investigación, lo que implica que un número considerable de investigadores quedan excluidos de los recursos que circulan por la red, de los que ellos mismos pudieran potencialmente aportar. Esto puede traer implícitamente una ineficiencia del potencial conectivo intra nodos y con redes externas, y con esto, del potencial de recursos que por la red pudieran circular.

3. Tantos los datos reticulares como estadísticos demuestran que no parece existir evidencia suficiente para sugerir relación alguna entre el cargo desempeñado por los investigadores y la posición dentro de la red. Parecerían exceptuarse de esta conclusión aquellos nodos cuyas relaciones de pertenencia al departamento son más esporádicas o irregulares (asociados y alumnos), sin embargo, no existe evidencia estadística suficiente como para confirmar esta excepción. 
REDES- Revista hispana para el análisis de redes sociales

Vol.14,\#5, J unio 2008

http: // revista-redes.rediris.es

4. De los agujeros estructurales que existen, los grupos intermediados son principalmente de investigadores externos, lo que entrega a los intermediadores un gran poder y control del flujo de información no redundante o distinta de la que se genera en los proyectos del departamento. La única intermediación "interna" (de un grupo de investigadores pertenecientes al departamento) es para un grupo de alumnos, los que se definen claramente como nodos receptores de recursos más que emisores de conocimiento, definiendo a este intermediador más como un "facilitador" de recursos, y con ello, un menor acceso a nueva información.

5. EI ARS permitió identificar claramente al grupo de investigadores principales de este departamento, sus principales áreas de investigación, y cómo estas se correlacionan con las más explotadas en el departamento, dando lugar a la posible existencia de lo que Crane (1972) definió como "colegio invisible". Sin embargo, también se pudo identificar a grupos secundarios de investigadores, y cómo estos también son capaces de posicionar una de sus áreas dentro de las principales a nivel global.

6. En síntesis, se puede hipotetizar que la posición académica no determina, ni menos asegura, un posicionamiento relevante y central en una red donde circulan intangibles tales como la información y el conocimiento. En otras palabras, el diferencial de acceso y generación de conocimiento e información en una red, que pudiera entregar el desempeñar determinados cargos académicos, no entrega un retorno acorde a lo que en términos jerárquicos cabría esperar.

\section{Bibliografía}

Ahuja, G. (2000). "Collaboration Networks, Structural Holes, and Innovation: A Longitudinal Study," Administrative Science Quarterly, 45, 425-455.

Arbonies, A.; Calzada, I. (2004). El poder del conocimiento tácito: por encima del aprendizaje organizacional. Intangible Capital, ISSN 1697-981, №. 6, 2004.

Arce C. y otros (2000). Recuperación de información métrica a partir de información nométrica con diseños de escalamiento multidimensional incompletos. Psicothema ISSN 0214 - 9915 CODEN PSOTEG 2000. Vol. 12, № 2, pp. 308-313

Arechavala, R. Díaz, C. (2001). Factores asociados con la productividad en la producción de ciencia y tecnología en contextos universitarios y en un centro de investigación. Universidad de Guadalajara.

Arias, A. (2003). Diferencias en el perfil de acumulación de capacidades tecnológicas en tres empresas mexicanas. El trimestre económico, ISSN 00413011, Vol. 70, №. 277, 2003, pags. 109-166.

Beckman, C; Secord, P. (1979). Social Psycology. New York u. a. 1979. 
REDES- Revista hispana para el análisis de redes sociales

Vol.14,\#5, J unio 2008

http: // revista-redes.rediris.es

Bolívar, A. (2000). Los centros educativos como organizaciones que aprenden. Madrid, La Muralla.

Burt, R. (2000). "Structural Holes versus Network Closure as Social Capital." University of Chicago and European d'Administration d'Affairs (INSEAD).

Camacho, M. y otros (2003). Técnicas de escalados multidimensionales aplicadas al fracaso del alumno en la asignatura de matemáticas empresariales. Facultad de Ciencias Económicas y Empresariales Departamento de Economía Aplicada III. Universidad de Sevilla.

Cantón, I. (2000). Las tecnologías como utopía en la sociedad de la información y el conocimiento y su incidencia en las instituciones educativas, en Lorenzo, M. et alt. (eds.) Las organizaciones educativas en la sociedad neoliberal, Granada: Grupo Editorial Universitario, 445-461

Caraballo, N. (2006). Gestión del Conocimiento: Aprendizaje individual versus aprendizaje organizativo. Intangible Capital, ISSN 1697-981, №. 13, 2006, pags. 308-326.

Casas, R. (2001). El enfoque de redes y flujos de conocimiento en el análisis de las relaciones entre ciencia, tecnología y sociedad. Instituto de investigaciones sociales, UNAM.

Coleman, J. (1990). Foundations of Social Theory. Cambridge, Mass.: Harvard University Press.

Cotillo-Pereira, Alberto (1994). Una teoría sociológica de la innovación en la ciencia: la obra del primer Mulkay. Dpto. de Sociología 1, Universidad Complutense, Madrid. Cristobal Torres, Elpio. de Sociología, UAM. Politica y Sociedad, 14/IS (1993-1994), Madrid» (PP. 115-142).

Crane, D. (1972). Invisible Colleges. Diffusion of Knowledge in Scientific Communities. The Chicago University Press.

Delgado, M. (2002). Los grupos en las organizaciones: delimitación conceptual y tipologías. ISSN 1131-6985, № 12, págs. 11-26.

Derek J. de Solla-Price (1961). Science Since Babylon. New Haven: Yale University Press.

Enguita, M. (2002). "La educación que queremos", Quinto ciclo de conferencias otoño 2002. Educación, economía y sociedad en España: Los desafíos del Trabajo en la Era Global, 29-79.

Flores, Margarita y Rello, Fernando (2003). "Capital social: virtudes y limitaciones" en Atria y otros (2003), Capital social y reducción de la pobreza en América Latina y el Caribe: en busca de un nuevo paradigma. CEPAL y Universidad del Estado de Michigan, Santiago de Chile.

Forni, P. y otros (2004). ¿Qué es el Capital Social y cómo Analizarlo en contextos de Exclusión Social y Pobreza? Julian Samora Research Institute. Michigan State University.

Gracia Guillén, Diego (2005). De los colegios invisibles al campus virtual. In: II Jornada Campus Virtual UCM: cómo integrar investigación y docencia en el CVUCM. Editorial Complutense, Madrid, pp. 12-18. ISBN 84-7491-787-5 
REDES- Revista hispana para el análisis de redes sociales

Vol.14,\#5, J unio 2008

http: // revista-redes.rediris.es

Gil, H. (2003). El aprendizaje interorganizativo en la Economía de la Información y el Conocimiento. Tesis Doctoral enmarcada en el proyecto de investigación SOCOTE financiada por el Plan Nacional y desarrollado en el seno del grupo de investigación ITIO. Universidad Politécnica de Valencia.

Granovetter, M. (1973). "The strength of weak ties", en American Journal of Sociology; vol 78, no 6. (pp. 1360 - 1380).

Julve, J. (2000). Implicaciones del Capital Social para la Ventaja Competitiva de la Empresa en un Contexto Evolutivo. Tesis doctoral dirigida por César Camisón Zornoza, Universitat Jaume I.

Knoke, D. (1990). Organizaing for collective action. The political economies of associations. Aldine de Gruyter. Nueva York.

Kruskal, J. (1964). Multidimensional scaling by optimizing goodness of fit to a nonmetric hypothesis, Psychometrika, 29, pp. 1-27.

Luhmann, Niklas (1984). Sistemas sociales. España: Universidad iberoamericana, Anthropos y Centro Editorial J averiano-Pontificia Universidad J averiana, 445 s..

Luhmann, Niklas (1995). Poder. España: Universidad I beroamericana y Anthropos, $177 \mathrm{s.}$

Luhmann, Niklas (1997). Organización y decisión. Autopoiesis, acción y entendimiento comunicativo. España: Universidad Iberoamericana y Anthropos, $138 \mathrm{~s}$.

Lundvall, B.A. (2000). "The learning Economy: Some implications for the knowledge base of health and education systems" en Knowledge Management in the Learning Society. Education and Skills, OCED, París, pp. 125-141.

Mitchell, J. (1969). Social Networks in Urban Situations. Manchester University Press: Manchester.

Molina, José Luis (2000). El organigrama informal en las organizaciones. Una aproximación desde el análisis de redes sociales. Revista Catalana de Sociologia, Nov. pág. 65-86.

Murillo A. y otros (2003). Optimización combinatoria en escalamiento multidimensional. 27 Congreso Nacional de Estadística e Investigación Operativa Lleida, 8- 11 de abril de 2003.

Mulkay, Michael J. (1972). "Conformityand Innovation inScience", The Sociological Review Monograph, vol. 18 (septiembre), pp. 5-23.

Nonaka, L.; Takeuchi, H. (1995). The Knowledge-Creating Company, Oxford University Press.

Nonaka, L.; Konno, N. (1998). "The Concept of "Ba": Building a Foundation for Knowledge Creation", California Management Review, 40, pp. 40-54.

Olazaran, Mikel; Lavía, Cristina y Beatriz Otero (2004) “¿Hacia una segunda transición en la ciencia? Política científica y grupos de investigación". Universidad del País Vasco. RES no 4 (2004) pp. 143-172

Peña, D. (2002). Análisis de Datos Multivariantes. Catedrático de Estadística. Universidad Carlos III Madrid. McGrawHill. ISBN: 84-481-3610-1.

Potocnjak, C. (2002). Construyendo capital social en la región de Aysen, Chile: hacia una interpretación del desarrollo como fenómeno conversacional. VII Congreso Internacional del CLAD sobre LA Reforma del Estado y de la Administración Pública. Lisboa, Portugal, 8-11 Octubre, 2002. 
REDES- Revista hispana para el análisis de redes sociales

Vol.14,\#5, J unio 2008

http: // revista-redes.rediris.es

Putnam, R. (1993). Making Democracy Work. New Jersey: Princeton University Press.

Revilla, E. (1996). Factores Determinantes del Aprendizaje Organizativo. Un Modelo de Desarrollo de Productos. Club Gestión de Calidad, Madrid.

Revilla, E. (1998). “De la Organización que Aprende Hacia la Gestión del Conocimiento", Comunicación presentada al VIII Congreso Nacional de ACEDE, Las Palmas de Gran Canaria.

Rosalba C. (2003). Intercambio y flujos de conocimiento en las redes. Itinerarios del conocimiento: formas dinámicas y contenido: un enfoque de redes / coord. por Matilde Luna Ledesma, 2003, ISBN 84-7658-673-6, pags. 306-354

Ruiz, A. (2004). Redes de Publicaciones de Académicos de Ingeniería. Un Análisis de la Respuesta Grupal en la Investigación. Departamento de Ingenierías, División de Estudios Profesionales. UNAM.

Sanz, Luis (2003). Análisis de redes sociales: o cómo representar las estructuras sociales subyacentes. Apuntes de Ciencia y Tecnología. Consejo Superior de Investigaciones Científicas (CSIC), Unidad de Políticas Comparadas (UPC).

Shea, G.P. y Guzzo, R.A. (1987). "Groups as Human Resources", Personnel and Human Resources Management, vol. 5, pp. 323-356.

Sundstrom, E.; de Meuse, K. P., y Futrell, D. (1990). «Work teams: Applications and effectiveness», American Psychologist, vol. 45, n.2, pp. 120-133.

Solís, C. (2004). Las Redes de Conocimiento en la Internacionalización de la investigación en administración. Depto. de Economía UAM. Sep. 2004, pags. 9-23.

Suárez, T. (2006). Los cuerpos académicos en la organización de las Universidades Públicas Mexicanas. Facultad de Contaduría y Administración. Ingenierías, AbrilJ unio 2006, Vol. IX, No. 31.

Uzzi, B. (1996). The sources and consequences of embeddedness for the economic performance of organizations: The network effect, American Sociological Review 61: 674-698.

Vargas, J. (2001). La Organización Aprendiente, Hitos de Ciencias Económico Administrativas, 19:33-40.

Zárraga, C.M. (2001). Factores determinantes y mecanismo de actuación para gestionar el conocimiento en equipos de trabajo. Vector plus: miscelánea científico - cultural, ISSN 1134-5306, №. 21, 2003, pags. 83-93.

Ziman, J. (1990), «Research as career». En Cozzens, S. E., Healey, P., Rip, A. y Ziman, J. (eds.), The Research System in Transition, Dordrecht, Kluwer Academic Publishers, pp: 345-359.

[1] Para efectos del presente artículo, hemos optado por no especificar una referencia directa al centro de investigación con el cual se elaboraron los datos y el consiguiente análisis de los mismos. Esta decisión, la hemos tomado con el fin de resguardar en todo momento la confidencialidad de la identidad de las personas que conformaron nuestro marco muestral.

[2] For effects of the present article, we opted to not specify a direct reference to the study department which the data was constructed. That is because we taken the options to protect in every moment the confidentiality of the identities of the people that conformed our sample.

[3] En Delgado (2002) y otros autores (Robbins, 1998; Guzzo y Dickson, 1996; Cohen y Bailey, 1997; Walton y Hackman, 1986; Alderfer, 1977) se coincide en definir a los grupos de trabajo como "un conjunto de individuos dependientes en sus tareas, que comparten responsabilidades, se ven y son vistos como una entidad social compacta, dentro de uno o más sistemas sociales superiores y que gestionan sus relaciones con otros grupos o individuos". A partir de esta definición se pueden identificar, 


\section{REDES- Revista hispana para el análisis de redes sociales \\ Vol.14,\#5, J unio 2008 \\ http: // revista-redes.rediris.es}

a su vez, grupos formales e informales (Walton y Hackman, 1986). Estos últimos tienen una función de socialización, y los formales la de resolución de problemas, y además, son los que conforman la espina dorsal de una organización como unidades formalmente reconocidas (Shea y Guzzo, 1987).

[4] Para efecto de esta ponencia nos hemos centrado en exponer dicho objetivo en virtud de la claridad, pertinencia y acotación de la exposición. Sin embargo, se hace notar que esta ponencia forma parte de una investigación más amplia, donde además se investigó la naturaleza y conformación de los grupos de investigación en la red; las áreas de conocimiento sobre las cuales se investiga; grupos que se erigen como "elites" académicas dentro de la red de investigación; el grado de relación de dependencia con grupos secundarios; y a partir de esto, las principales áreas de investigación que se explotan en el departamento. Dejamos pendientes dichos objetivos como referencias para futuras comunicaciones.

[5] Los nuevos colegios invisibles pueden ser redes o grupos sociales que se identifican por ser caminos distintos a los de las redes de citas o coautorías, ya que son anteriores a ellas.

[6] Entenderemos conectividad como la existencia de un camino que une a dos científicos en una dirección determinada, independientemente de su longitud. El énfasis de esta aproximación se centra en el establecimiento de una red total en la cual se hayan insertos los investigadores, sean concientes o no, donde con un cálculo de la conectividad existente se determinará la existencia de "colegios invisibles" (Molina 2000).

[7] El concepto de Capital Social es profusamente utilizado en ciencias sociales desde la década del noventa, a partir de las contribuciones de autores como Bourdieu, Coleman, Putnam y Portes, y constituye probablemente una de las innovaciones más prometedoras de la teoría social contemporánea. Bourdieu puede ser considerado el primer autor que realizó un estudio sistematizado sobre el capital social, centrándose en el análisis de los beneficios que obtienen los individuos a partir de su participación en determinados grupos y en la construcción de relaciones sociales con el mero objetivo de crear este tipo de capital. De este modo, lo define como "el agregado de los recursos reales o potenciales que se vinculan con la posesión de una red duradera de relaciones más o menos institucionalizadas de conocimiento o reconocimiento mutuo"... "De allí que, a través del capital social, los actores puedan obtener acceso directo a recursos económicos (préstamos subsidiarios, información sobre inversiones, mercados protegidos); pueden incrementar su capital cultural gracias a los contactos con expertos o individuos refinados, o de manera alternativa, asociarse a instituciones que otorgan credenciales valoradas" (FORNI, P. et al. 2004).

[8] En cuanto a las argumentaciones sobre la densidad de las relaciones en un determinado grupo, estamos haciendo clara alusión a los parámetros teóricos desarrollados y personificados en la figura de Putnam, los cuales, en términos generales, se resumen en entender el capital social como "aspectos de la organización social tales como confianza, normas y redes, que pueden mejorar la eficiencia de una sociedad al facilitar la acción coordinada" (PUTNAM, R. 1993). En consecuencia, el eje central en la obra de Putnam gira en torno a comprender los problemas que presenta la acción colectiva, específicamente trata sobre la tendencia comunitaria hacia la cooperación en pos del bien común o al oportunismo, y los factores que condicionan la primera o la segunda elección, lo que a su vez, genera ciertas consecuencias en el desarrollo económico y democrático- institucional de las comunidades.

[9] Un agujero estructural, o "huecos estructurales", hace referencia a características estructurales de las redes, específicamente cuando dentro de una red existen diversas zonas densas unidas unas a otras por escasos lazos y/o por algún(os) nodo(s). Estos actores alcanzan una posición central entre los actores de la red, especialmente por las ventajas de acceso a la información y conocimientos que puedan circular por la red, y particularmente de estos núcleos densos de nodos. En términos de redes de conocimiento, estas posiciones se correlacionan con actividades innovadoras y competitivas, sin embargo, estos actores contarán con menor capital social integrador que aquellos que operan en las zonas más densas. (AHUJ A, 2000).

[10] En cuanto a las condiciones metodológicas particulares del estudio, se define como una investigación de tipo transversal, ya que la recogida y análisis de datos se circunscriben al periodo comprendido entre Febrero del 2004 y Junio del 2006. Debe considerarse que algunos de los proyectos analizados aún no finalizan, por lo tanto, se han considerado los investigadores que hasta la fecha mencionada comprendían los equipos de trabajo. Por último, las unidades de observación escogidas para la investigación no fueron seleccionadas al azar, representan a la totalidad del universo en estudio, y como no se busca manipular variable de control alguna, este estudio toma un carácter no experimental. [11] Como se puede observar, realizar un análisis estadístico para categorías con grados de libertad tan bajos resultaría improcedente. Por tal motivo, se propone reducir las categorías profesionales en función de autonomía investigadora, relación contractual y carga de trabajo en el departamento. En este sentido, se conformaron 3 categorías: a) Profesor = Catedrático + Titular + Ayudante Doctor, b) Becario = Becario + Alumno, c) asociado.

[12] En forma complementaria a este análisis se calculó el coeficiente "Eta" apropiado para la medición de asociación entre una variable dependiente escalar y una variable independiente nominal, en este caso la agrupación de cargo de profesores. El valor de Eta es de 0,203 valor tan cercano a cero que reafirma que la centralidad no es significativamente distinta para las agrupaciones de cargos.

[13] De la misma manera, al igual que en el caso del análisis de centralidad de grado se calculó el coeficiente Eta para la centralidad de intermediación obteniéndose como resultado un valor de 0,134 valor tan cercano a cero que reafirmar la no existencia de diferencias en dicho tipo de centralidad a partir de las categorías de cargo agrupadas.

[14] a) Grupo 1: Profesor (Catedrático + Titular + Ayudante Doctor) b); Grupo 2: Asociado-Becario (Becario + Alumno) 